\title{
Polimorfismos del gen del receptor de muerte celular programada 1 (PDCD1) y diabetes tipo 1 en población chilena
}

\author{
SYLVIA FLORES R. ${ }^{\text {la }}$, MEGAN BEEMS ${ }^{1 \mathrm{~b}}$, AMAYA OYARZÚN A. ${ }^{\text {lc }}$, \\ ELENA CARRASCO P. ${ }^{2 \mathrm{~d}}$, FRANCISCO PÉREZ B. ${ }^{\text {lac }}$
}

\section{Programmed cell death 1 (PDCD1) gene polymorphisms and type 1 diabetes in Chilean children}

Background: Programmed cell death 1 (PDCD-1) immune-receptor is a key element in the negative regulation of peripheral tolerance in $T$ cells. Several polymorphisms of this gene have been described and it is linked with susceptibility to autoimmune diseases like Lupus and Multiple Sclerosis. Aim: To analyze four gene polymorphisms of PDCD-1 gene and explore its possible contribution as a susceptibility gene for type 1 diabetes (T1D). Patients and Methods: We analyzed 160 cases with T1D of recent diagnosis aged $9.5 \pm 3.3$ years and 160 control children aged 10.7 \pm 3.1 years. Four genetic variants of PDCD-1 gene were studied (PD1.2; PD1.5; PD1.6 and PD1.9) by polymerase chain reaction and restriction enzymes. Autoantibodies GAD65 and anti-IA-2 were also measured in all studied children. The comparison of allelic and genotypic frequency and consistency with respect to Hardy-Weinberg equilibrium test were analyzed using Chi-square and Fisher exact test. Results: No differences between cases and controls were observed for PDCD1.2; PDCD1.5 and PDCD1.9 polymorphisms. PDCD1.6 polymorphism (carriers of allele A) had a higher frequency in the control group (0.794 versus $0.644, p<0.017)$. There was no particular association of these polymorphisms with anti- GAD65 and anti-IA-2 antibodies among patients with T1D. Conclusions: Only PDCD1.6 polymorphism showed differences between T1D cases and controls. Possibly, none of these genetic variants of PDCD1 has a relevant role as a marker for T1D in the Chilean population.

(Rev Med Chile 2010; 138: 543-550).

Key words: Diabetes mellitus; Genetic; Human; PDCD-1 protein; Polymorphisms; Type 1.

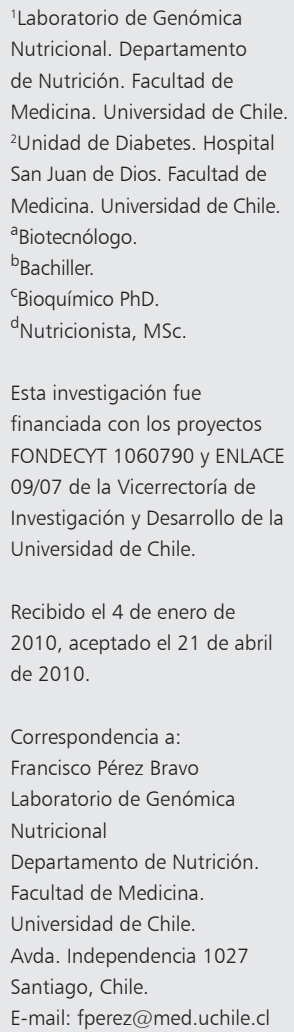

L a diabetes mellitus tipo 1 (DM1) constituye el trastorno endocrino-metabólico más frecuente en la infancia y adolescencia, caracterizado por la incapacidad del páncreas de producir insulina, lo que confiere a esta enfermedad características únicas entre las patologías crónicas infantiles. Su etiología es extremadamente compleja, donde intervienen factores ambientales que interaccionan con componentes de predisposición genética que desembocan en un irreversible ataque de origen autoinmune contra las células productoras de insulina, ubicadas en el islote pancreático $^{1}$. Dentro de los factores ambientales figuran infecciones virales ${ }^{2,3}$, toxinas y $\operatorname{dieta}^{4}$, entre otros, conduciendo de alguna manera aún desconocida a la ruptura de la tolerancia autogénica normal.

En relación a los factores genéticos, el sistema mayor de histocompatibilidad (MHC) es el candi- 
dato principal, desde hace tres décadas, debido al papel central que juega justamente en el proceso de tolerancia autogénica. Sin embargo, no constituye un marcador absoluto de la enfermedad, lo que ha generado una intensa búsqueda de nuevos genes o regiones génicas candidatas ${ }^{5-7}$.

Actualmente, la búsqueda de genes candidatos está dirigida hacia el descubrimiento de nuevas regiones génicas de susceptibilidad a DM1. En este sentido, se han identificado dos regiones que son objeto de amplias investigaciones. La variante $\mathrm{PTPN}_{22}$ del gen que codifica para la proteína tirosina-fosfatasa (LYP) como nuevo factor de susceptibilidad a DM1 actuando también a través de la regulación de la respuesta del linfocito $\mathrm{T}^{8}$ y los genes que codifican para los receptores de muerte celular programada 1 (PDCD1) dada su influencia en el reconocimiento de estructuras que regulan negativamente la relación entre la célula presentadora del antígeno (APC) y el linfocito $\mathrm{T}^{9}$.

Las moléculas de muerte celular programada (PDCD1) pertenecen a la familia de receptores CD28 y son expresadas en las células activadas T, B $y$ mieloide ${ }^{10}$. Se ha postulado que PDCD1 juega un importante rol en la inducción y/o mantención de la tolerancia periférica. Por medio de la interacción con sus ligandos (PDL1 y PDL2), PD-1 inhibe la estimulación antigénica de las células $\mathrm{T}$ y $\mathrm{B}$. Los ligandos $\mathrm{PD}$-Ls pertenecen a la familia $\mathrm{B} 7 \mathrm{y}$ pueden ser expresados tanto en órganos linfoides, como no linfoides. El balance de señales inhibitorias (PDCD1 y CTLA-4) y de estimulación (B7) provee a las interacciones en la superficie celular de los linfocitos $\mathrm{T}$ y células presentadoras de antígenos de los mecanismos cruciales de la homeostasis celular para el mantenimiento de la tolerancia periférica ${ }^{11}$. La disrupción de este delicado balance puede conducir al quiebre de la auto-tolerancia y por lo tanto generar un desbalance conducente a la patogénesis de las enfermedades autoinmunes.

El gen humano para PDCD1 está localizado en el cromosoma 2q37 y se han identificado varios polimorfismos de nucleótido simple (SNPs) asociados con desórdenes autoinmunes tales como: lupus eritematoso sistémico (LES), artritis reumatoidea (AR), tiroiditis y DM1 entre otras ${ }^{12,13}$. En el caso de LES, los estudios realizados en Suecia muestran asociación del polimorfismo PDCD1 con manifestaciones renales del LES ${ }^{14}$. También se ha descrito asociación en población China ${ }^{15}$. Estudios en Taiwán muestran una asociación po- sitiva ${ }^{16}$ y otra negativa para $\mathrm{LES}^{17}$. Este fenómeno también se observó en una cohorte poblacional multiétnica conformada por caucásicos, asiáticos, hispano y afroamericanos en Estados Unidos de Norteamérica (USA) ${ }^{18}$ y en un estudio multi-poblacional europeo ${ }^{19}$, efecto similar se ha observado en población mexicana ${ }^{20}$. El estudio realizado en población española, mostró resultados contradictorios $^{21,22}$. En AR, los hallazgos son similares en cuanto a asociación positiva en población de Taiwán ${ }^{23}$, y negativa en japoneses ${ }^{24}$. También se han descrito otras asociaciones de estos polimorfismos con: espondilitis anquilosante en coreanos ${ }^{25}$, encefalomielitis autoinmune ${ }^{26}$, enfermedad hepática autoinmune ${ }^{27,28}$ y cirrosis biliar ${ }^{29}$.

En ratas NOD el polimorfismo en $P D C D 1$ y su ligando PD-L1 se asocia con diabetes autoinmu$n \mathrm{e}^{30}$. El modelo knock out para PDCD1 ${ }^{-1-}$ mostró una fuerte polarización hacia la respuesta Th1, autoinmunidad acelerada e infiltración de islotes ${ }^{31}$. Los estudios realizados en pacientes con DM1 han mostrado resultados negativos en población francesa ${ }^{32} y$ otros que sugieren un papel positivo del gen PDCD1 con susceptibilidad a la DM1 en población danesa ${ }^{33}$ y japonesa ${ }^{34,35}$. El propósito de este estudio fue analizar cuatro polimorfismos del gen $P D C D-1$ y explorar su posible contribución como gen de susceptibilidad a la diabetes tipo 1 (DM1) en la población chilena.

\section{Pacientes y Métodos}

Los casos con DM1 correspondieron a niños y adolescentes menores de 15 años con diagnóstico reciente de la enfermedad (definido como evento ocurrido en las 3 semanas previas al ingreso al estudio), provenientes de diversos centros hospitalarios de la Región Metropolitana (Hospitales San Borja-Arriarán, Exequiel González Cortés y San Juan de Dios). Ciento sesenta pacientes con DM1 fueron incorporados al estudio. El grupo control estuvo formado por 160 niños aparentemente sanos, sin antecedentes familiares de diabetes $\mathrm{u}$ otras enfermedades autoinmunes (datos obtenidos mediante encuesta familiar). Las características clínicas de ambos grupos de niños se presentan en la Tabla 1. Los niños controles fueron contactados de tres colegios de Santiago (comunas de Quinta Normal, Independencia y Macul), fueron incorporados al azar en un llamado abierto y voluntario realizado en cada colegio por 
Polimorfismos PDCD1 y diabetes tipo 1 en población chilena - S. Flores R. et al

Tabla 1. Características clínicas e inmunológicas de los grupos de estudio

\begin{tabular}{|lcc|}
\hline & $\begin{array}{c}\text { Casos } \\
(\mathbf{n = 1 6 0 )}\end{array}$ & $\begin{array}{c}\text { Controles } \\
(\mathbf{n}=\mathbf{1 6 0})\end{array}$ \\
\hline Edad (años) & $9,5 \pm 3,3$ & $10,7 \pm 3,1$ \\
Hombre/ Mujer & $68 / 92$ & $87 / 73$ \\
$\begin{array}{l}\text { Edad de Diagnóstico } \\
\text { (años) }\end{array}$ & $8,2 \pm 3,6$ & - \\
\hline $\begin{array}{l}\text { Total de anticuerpos } \\
\text { positivos (\%) }\end{array}$ & 73,4 & 1,25 \\
\hline Anti.GAD65 (\%) & 82,5 & 1,25 \\
Anti-IA-2 (\%) & 64,3 & 0,00 \\
\hline $\begin{array}{l}\text { Enfermedad } \\
\text { autoinmune (\%) }\end{array}$ & 4,4 & 1,9 \\
\hline
\end{tabular}

intermedio de los centros de padres respectivos por lo que estimamos que son comparables en términos de su estatus socio-genético respecto de los casos con DM1. En todos los casos y controles se analizó la frecuencia de cuatro polimorfismos para el gen $P D C D 1$ y un perfil de auto-anticuerpos anti-GAD65 y anti-IA-2. El protocolo de esta investigación fue aprobado por los comités de ética respectivos y todos los pacientes, controles o sus padres o tutores entregaron su consentimiento por escrito.

\section{Extracción de ADN y determinación de} polimorfismos PDCD1

El ADN genómico se extrajo a partir de una muestra de sangre periférica $(3 \mathrm{ml})$ utilizando técnicas estándares de lisis celular (Winkler, Santiago,
Chile). Los polimorfismos PDCD1 se determinaron mediante la utilización de partidores específicos de acuerdo a la Tabla 2. Cada reacción de PCR fue realizada en $25 \mu \mathrm{L}$ de solución conteniendo 5 pmoles de cada par de partidores, $50 \mathrm{ng}$ de ADN de cada una de las muestras, $200 \mu \mathrm{M}$ de cada dNTP y 0,5 U de Taq-Polimerasa. La reacción de PCR se estandarizó en un período de 3 minutos a $95^{\circ} \mathrm{C}$ para la activación de la enzima, seguido de 35 ciclos que consistieron en un período de denaturación del $\mathrm{ADN}$ a $95^{\circ} \mathrm{C}$ por 15 segundos, un período de unión de los partidores a $64^{\circ} \mathrm{C}(\mathrm{PD} 1,5 ; \mathrm{PD} 1,2)$, $54^{\circ} \mathrm{C}(\mathrm{PD} 1,9), 56^{\circ} \mathrm{C}(\mathrm{PD} 1,6)$ y por 15 segundos y un período de extensión de la polimerasa a $72^{\circ} \mathrm{C}$ por 15 segundos. Para realizar el PCR se ocupo un Termociclador Biometra ${ }^{\mathrm{R}}$ (Alemania). Para llevar a cabo el análisis mediante fragmentos de restricción (RFLP) se tomaron $6 \mu \mathrm{L}$ de cada producto de PCR que fue incubado a $37^{\circ} \mathrm{C}$ durante 1 hora con $0,5 \mathrm{U}$ de la endonucleasa de restricción apropiada para cada polimorfismo. El producto de digestión fue visualizado en gel de agarosa al $2 \%$.

\section{Análisis serológico de anticuerpos}

Los anticuerpos anti-GAD65 y anti-IA2 fueron determinados mediante inmunoensayo enzimático (ELISA) con el uso de kits comerciales (Medizym ${ }^{\circledR}$ Diagnostic, Berlin, Alemania). La detección de anticuerpos se llevó a cabo de manera semi-cuantitativa tomando como referencia el valor de $5 \mathrm{UI} / \mathrm{mL}$ para GAD65 y $10 \mathrm{UI} / \mathrm{mL}$ para IA2. Aquellas muestras con un título mayor o igual a dicho valor fueron consideradas muestras positivas. Se obtuvo una sensibilidad y especificidad del $92,3 \%$ y $98,6 \%$ para anti-GAD 65 y de $75 \%$ y $98 \%$ para anti-IA-2. Todos los valores se encuentran

Tabla 2. Método PCR-RFLP, partidores PCR y enzimas de restricción (RE) utilizados en el estudio molecular del gen PDCD-1

\begin{tabular}{|c|c|c|c|c|c|}
\hline & SNP & Ubicación & Método & RE & Partidores \\
\hline PD 1.2 & $6438 \mathrm{G} / \mathrm{A}$ & Intrón 2 & PCR-RFLP & Msp I & $\begin{array}{l}\text { F: 5'-AGCGGCACCTACCTCTGTGG-3`} \\
\text { R: } 5{ }^{\prime}-\text { GTGGGCTGTGGGCACTTCTG-3`}\end{array}$ \\
\hline PD 1.5 & $7785 \mathrm{C} / \mathrm{T}$ & Exón 5 & PCR-RFLP & Alu I & $\begin{array}{l}\text { F: } 5^{\prime} \text { - AGACGGAGTATGCCACCATT-3’ } \\
\text { R: } 5^{\prime} \text { - CACTGTGGGATTGAGACAT-3’ }\end{array}$ \\
\hline PD 1.6 & 8737 G/A & 3'UTR & PCR-RFLP & Nla III & $\begin{array}{l}\text { F: } 5^{\prime} \text { - TCAGAAGAGCTCCTGGCTGT-3’ } \\
\text { R: 5'- GGGGAACGCCTGTACCTT-3’' }\end{array}$ \\
\hline PD 1.9 & $7625 \mathrm{C} / \mathrm{T}$ & Exón 5 & PCR-RFLP & Bpu 101 & $\begin{array}{l}\text { F: } 5^{`} \text { - GGACAGCTCAGGGTAAGCAG-3`} \\
\text { R: } 5^{`}-\text { AGGGTCTGCAGAACACTGGT-3`}\end{array}$ \\
\hline
\end{tabular}


dentro de los rangos esperados para un ELISA de acuerdo al programa DASP (Diabetes Antibody Standardization Program $)^{36}$.

Estadística: Tanto el análisis de las frecuencias alélicas y genotípicas en el estudio caso-control, como el porcentaje de autoanticuerpos entre los pacientes diabéticos y el grupo control se analizaron con la prueba estadística de $\chi^{2}$ mediante el software SHEsis, el cual también se utilizó para determinar el equilibrio de Hardy-Weinberg en el estudio caso-control ( $\mathrm{p}$ value $<0,05$ fue considerado como estadísticamente significativo).

\section{Resultados}

La Tabla 3 muestra la distribución de todos los polimorfismos $P D C D 1$ analizados en este estudio. El análisis del polimorfismo PDCD1.2 no mostró diferencias relevantes al comparar pacientes con DM1 y controles. La frecuencia de la combinación A/A para esta variante genética fue prácticamente nula en la población analizada. Además, la distribución de este polimorfismo no se encontró en equilibrio de Hardy-Weinberg. En relación al polimorfismo PDCD1.5 de acuerdo al p-value global, se observaron diferencias significativas al

Tabla 3. Distribución de frecuencias genotípicas y alélicas PDCD1 en ambos grupos

\begin{tabular}{|c|c|c|c|c|c|c|c|}
\hline \multirow[t]{2}{*}{ Polimorfismo } & \multicolumn{2}{|c|}{ DM1 } & \multicolumn{2}{|c|}{ Control } & \multirow[t]{2}{*}{ OR } & \multirow[t]{2}{*}{ IC 95\% } & \multirow[t]{2}{*}{ p-value } \\
\hline & $\mathbf{n}$ & Frec. & $\mathbf{n}$ & Frec. & & & \\
\hline \multicolumn{8}{|l|}{ PDCD 1.2} \\
\hline A/A & 1 & 0,060 & 1 & 0,060 & & & \\
\hline $\mathrm{A} / \mathrm{G}$ & 69 & 0,431 & 81 & 0,506 & & & 0,403 \\
\hline $\mathrm{G} / \mathrm{G}$ & 90 & 0,562 & 78 & 0,487 & & & \\
\hline$\%$ alelo $\mathrm{A}$ & & 22,2 & & 25,9 & 0,814 & $0,566-1,170$ & 0,267 \\
\hline$\%$ alelo G & & 77,8 & & 74,1 & & & \\
\hline \multicolumn{8}{|l|}{ PDCD 1.5} \\
\hline$C / C$ & 27 & 0,169 & 37 & 0,231 & & & \\
\hline$C / T$ & 129 & 0,806 & 107 & 0,669 & & & 0,004 \\
\hline $\mathrm{T} / \mathrm{T}$ & 4 & 0,025 & 16 & 0,100 & & & \\
\hline$\%$ alelo C & & 57,2 & & 56,6 & 1,036 & $0.750-1.402$ & 0,873 \\
\hline \% alelo T & & 42,8 & & 43,4 & & & \\
\hline \multicolumn{8}{|l|}{ PDCD 1.6} \\
\hline A/A & 32 & 0,200 & 25 & 0,156 & & & \\
\hline$A / G$ & 71 & 0,444 & 102 & 0,637 & & & 0,002 \\
\hline $\mathrm{G} / \mathrm{G}$ & 57 & 0,356 & 33 & 0,206 & & & \\
\hline$\%$ alelo $\mathrm{A}$ & & 42,2 & & 47,5 & 0,806 & $0,590-1,101$ & 0,176 \\
\hline$\%$ alelo G & & 57,8 & & 52,5 & & & \\
\hline \multicolumn{8}{|l|}{ PDCD 1.9} \\
\hline$T / T$ & & 0 & & 0 & & & \\
\hline $\mathrm{T} / \mathrm{C}$ & 133 & 0,831 & 132 & 0,825 & & & 0,882 \\
\hline C/C & 27 & 0,169 & 28 & 0,175 & & & \\
\hline$\%$ alelo T & & 58,4 & & 58,8 & 0,987 & $0,720-1,352$ & 0,936 \\
\hline$\%$ alelo C & & 41,6 & & 41,2 & & & \\
\hline
\end{tabular}


Polimorfismos PDCD1 y diabetes tipo 1 en población chilena - S. Flores R. et al

Tabla 4. Frecuencias haplotípicas relevantes entre casos con DM1 y controles para los polimorfismos PD 1.2; PD 1.5; PD 1.6 y PD 1.9 del gen de PDCD-1

\begin{tabular}{|lcccc|}
\hline Haplotipos & $\begin{array}{c}\text { Frecuencia casos } \\
(\mathbf{\%})\end{array}$ & $\begin{array}{c}\text { Frecuencia controles } \\
\mathbf{( \% )}\end{array}$ & OR & 95 \% IC \\
CCGA & 12,2 & 7,1 & 1,859 & $1,080-3,202$ \\
CCGG & 27,3 & 26,5 & 1,069 & $0,752-1,521$ \\
CTAA & 2,2 & 8,4 & 0,250 & $0,107-0,582$ \\
CTGA & 6,0 & 4,7 & 1,319 & $0,658-2,641$ \\
CTGG & 7,2 & 9,1 & 0,790 & $0,447-1,398$ \\
TCGA & 0,5 & 3,5 & 0,134 & $0,025-0,733$ \\
\hline TCGG & 0,1 & 4,1 & 0,028 & $0,003-0,244$ \\
TTAA & 14,9 & 14,4 & 1,066 & $0,687-1,656$ \\
\hline TTGA & 4,9 & 9,3 & 0,514 & $0,273-0,966$ \\
TTGG & 19,6 & 9,6 & 2,362 & $1,485-3,758$ \\
\hline
\end{tabular}

No se consideraron frecuencias inferiores a 0,03 en DM1 y controles.

comparar casos con DM1 y controles $(\mathrm{p}=0,004)$, con una mayor frecuencia del genotipo $\mathrm{C} / \mathrm{C}$ en controles $(0,231$ versus 0,169$)$.

El polimorfismo PDCD1.6 mostró diferencias significativas al comparar casos con DM1 y controles, indicando una posible asociación del genotipo G/G con DM1 (0,356 versus $0,206, \mathrm{p}=$ $0,004)$. Finalmente, el polimorfismo PDCD1.9 no mostró diferencias significativas al comparar casos con DM1 y controles, la frecuencia del genotipo $\mathrm{T} / \mathrm{T}$ fue nula en los dos grupos y esta variante no se encontró en equilibrio de Hardy-Weinberg.

Los resultados de la Tabla 4 muestra que los haplotipos CCGA $(12,2 \%$; OR $=1,859)$ y TTGG $(19,6 \% ; O R=2,362)$ son bastante más frecuentes en la población con DM1 generando los más altos OR, en ambos casos estos haplotipos podrían llegar a constituirse en marcadores de predisposición para la enfermedad. Las combinaciones haplotípicas TTGA, TCGA y TCGG mostraron los valores más bajos de OR, baja frecuencia en el grupo con DM1 y por lo que podrían plantearse como posibles haplotipos de protección.

\section{Discusión}

La DM1 es una enfermedad en la que interactúan factores genéticos y ambientales. Desde el punto de vista inmunológico ha sido descrita como eminentemente autoinmune, aunque los mecanismos de origen apoptótico cada día adquieren mayor relevancia en su etiología. Desde el punto de vista de la distribución de los auto-anticuerpos, nuestros resultados mostraron que más de la mitad de los pacientes diabéticos estudiados presentaron al menos uno de los dos anticuerpos cuantificados (GAD65 e IA-2), mientras que en el grupo control la presencia de dichos anticuerpos fue prácticamente nula. Estos resultados no son sorprendentes, puesto que desde hace tiempo se conoce que la presencia de una batería de anticuerpos como: GAD65, IA2 e IAA se manifiestan en el inicio de la DM1 y se han asociado con un mayor riesgo para desarrollar esta patología ${ }^{36}$.

Dado el importante rol que manifiestan tanto la molécula PD-1 como CTLA-4 en la regulación negativa de la proliferación de células $\mathrm{T}$, estos inmuno-receptores han sido objeto de innumerables estudios con el fin de establecer su relación con patologías de origen autoinmune $e^{12,37}$. Considerando que estas proteínas son una señal muy relevante en los procesos de regulación negativa de las células $T^{11}$, se han realizado múltiples análisis genéticos que evidencian la asociación de varios polimorfismos en las proximidades del gen $P D C D$ $1^{38}$ dentro de los cuales figuran los polimorfismos PDCD 1.2, PDCD 1.5, PDCD 1.6, PDCD 1.9, como locus importantes de asociación con enfermedades autoinmunes, y en particular con DM1 en distintas poblaciones a nivel mundial. 
En este sentido, nuestro estudio mostró que los polimorfismos PDCD 1.5 y PDCD 1.6 tienen una asociación cruda mayor que los polimorfismos PDCD 1.2 y PDCD 1.9 con DM1. Sin embargo, ningún polimorfismo en forma individual alcanzó un nivel de relevancia como para ser considerado un marcador genético de la DM1. Lo anterior, reafirma una vez más el hecho de que los factores de riesgo genéticos descritos hasta la fecha en la DM1 no son absolutos, se distribuyen heterogéneamente entre las distintas poblaciones, probablemente debido a la activa participación de factores ambientales en la etiología de la enfermedad ${ }^{3,4}$.

En base a los análisis realizados en este estudio, podemos decir que nuestros resultados genéticos se acercan más a los obtenidos en población europea que a los observados en población asiática, lo cual guardaría cierta concordancia con los datos de composición poblacional actual que indican que nuestra población esta compuesta principalmente por individuos de ascendencia caucásica. No obstante, se debe considerar que el tamaño muestral es aún pequeño para realizar consideraciones mayores. Los datos de frecuencias polimórficas para el gen $P D C D 1$ analizadas en este estudio no mostraron una especial distribución en la población con DM1, sugiriendo que este marcador de susceptibilidad tiene un efecto nulo o muy pequeño en nuestra población.

Existen evidencias que establecen que el gen $P D C D 1$ y sus moléculas ligando son un eficiente regulador negativo de la activación del linfocito $\mathrm{T}^{13}$, además se ha planteado que esta molécula o sus ligandos podrían ser considerados como un marcador de "agotamiento" de células T, es decir, perdida de regulación negativa, hiperactivación y alteración apoptótica de la célula T alterada ${ }^{39}$. Se ha observado que al bloquear la interacción del receptor PD-1 con su ligando PD-L1 existe una reversión del fenotipo "agotado". Esto se llevo a cabo administrando anticuerpos anti-PD-L1, lo que resultó en la reactivación de las células $\mathrm{T}$, aumentando la producción de citoquinas, su proliferación y su actividad citotóxicas ${ }^{39}$.

Recientemente, se ha demostrado que alteraciones intestinales estarían asociadas a pacientes con DM1 o a individuos en riesgo de desarrollar ésta enfermedad. Esta nueva forma de visualizar la enfermedad le da un énfasis particular a las células $\mathrm{T}$, las cuales cumplirían un papel central en el equilibrio de tolerancia. La concepción de un "intestino perforado" dentro del marco de esta patología permitiría una mayor exposición del sistema inmune intestinal a ciertos antígenos comunes, que en presencia de una microbiota alterada reduciría la auto-tolerancia mediada por las células T reguladoras ${ }^{40,41}$. En este nuevo escenario, los estudios dirigidos a la caracterización de polimorfismos funcionales en la célula $\mathrm{T}$ adquieren un a relevancia fundamental.

Agradecimientos: Los autores agradecen la colaboración de todos los niños y sus familias participantes en este estudio. A los Hospitales San Juan de Dios, San Borja-Arriarán y a la Fundación de Diabetes Juvenil de Chile.

\section{Referencias}

1. Anjos S, Polychronakos C. Mechanisms of genetic susceptibility to type 1 diabetes: beyond HLA. Mol Gen and Metab 2004; 81: 187-95.

2. Bach JF. Infections and autoimmune diseases. J Autoimmun 2005; 25: 74-80.

3. Hyoty H. Enterovirus infections and type 1 diabetes. Ann Med 2002; 34: 138-47.

4. Myers MA, Hettiarachchi KD, Ludeman JP, Wilson AJ, Wilson CR, Zimmet PZ. Dietary microbial toxins and type 1 diabetes. Ann NY Acad Sci 2003; 1005: 418-22.

5. Devendra D, Eisenbarth GS. Immunologic endocrine disorders. J Allergy Clin Immunol 2003; 111: 624-36.

6. Gregersen PK, Behrens TW. Genetics of autoimmune diseases-disorders of immune homeostasis. Nat Rev 2006; 7: 917-28.

7. Alizadeh BZ, Koeleman BP. Genetic polymorphisms in susceptibility to type 1 diabetes. Clin Chim Acta 2008; 387: 9-17.

8. Bottini N, Musumeci L, Alonso A, Rahmouni S, Nika K, Rostamkhani $\mathrm{M}$, et al. A functional variant of lymphoid tyrosine phosphatase is associated with type 1 diabetes. Nat Genet 2004; 36: 337-8.

9. Vang T, Miletic AV, Arimura Y, Tautz L, Rickert RC, Mustelin T. Protein tyrosine phosphatases in autoimmunity. Ann Rev Autoimmunity 2008; 26: 29-55.

10. Keir ME, Butte MJ, Freeman GJ, Sharpe AH. PD-1 and its ligands in tolerance and immunity. Ann Rev Immunol 2008; 26: 677-704.

11. Sharpe AH, Wherry EJ, Ahmed R, Freeman GJ. The function of programmed cell death 1 and its ligands in regulatin autoimmunity and infection. Nat Immunol 2007; 8: 239-45.

12. Okazaki T, Honjo T. PD-1 and PD-1 ligands: from 
discovery to clinical application. Int Immunol 2007; 19: 813-24.

13. Okazaki T, Honjo T. The PD-1-PD-L pathway in immunological tolerance. Trends in immunology 2006; 27(4).

14. Johansson M, Arlestig L, Moller B, Rantapää-Dahlqvist S. Association of a PDCD1 polymorphism with renal manifestations in systemic lupus erythematosus. Arthritis \& Rheumatism 2005; 52: 1665-9.

15. Wang Q, Ye D, Yin J, Zhang G, Zhang Y, Zhang X. Programmed cell death 1 genotypes are associated with susceptibility to systemic lupus erythomatosus among Chinese. Arch Dermatol Res 2007; 300: 91-3.

16. Wang SC, Chen YJ, Ou TT, Wu CC, Tsai WC, Liu HW, et al. Programmed death-1 gene polymorphisms in patients with systemic lupus erythematosus in Taiwan. J Clin Immunol 2006; 26: 506-11.

17. Lin SC, Yen JH, Tsai JJ, Tsai WC, Ou TT, Liu HW, et al. Association of a programmed death 1 gene polymorphism with the development of rheumatoid arthritis, but not systemic lupus erythematosus. Arthritis \& Rheumatism 2004; 50: 770-5.

18. Thorburn CM, Prokunina-Olsson L, Sterba KA, Lum RF, Seldin MF, Alarcón-Riquelme ME, et al. Association of PDCD1 genetic variation with risk and clinical manifestations of systemic lupus erythematosus in a multiethnic cohort. Genes Immunol 2007; 8: 279-87.

19. Prokunina L, Castillejo-López C, Oberg F, Gunnarsson $\mathrm{I}$, Berg L, et al. A regulatory polymorphism in PDCD1 is associated with susceptibility to systemic lupus erythematosus in humans. Nat Genet 2002; 32: 666-70.

20. Velázquez-Cruz R, Orozco L, Espinosa-Rosales F, Carreño-Manjarrez R, Solís-Vallejo E, et al. Association of PDCD1 polymorphisms with chilhood-onset systemic lupus erythematosus. Eur J Hum Genet 2007; 15: 33641.

21. Ferreiros-Vidal I, Gómez-Reino JJ, Barros F, Carracedo A, Carreira P, González-Escribano, et al. Association of PDCD1 with susceptibility to systemic lupus erythematosus. Arthritis \& Rheumatism 2004; 50: 2590-7.

22. Ferreiros-Vidal I, D'Alfonso S, Papasteriades C, Skopouli FN, Marchini M, Scorza R, et al. Bias in association studies of systemic lupus erythematosus susceptibility due to geographical variation in the frequency of a programmed cell death 1 polymorphism across Europe. Genes Immunol 2007; 8: 138-46.

23. Kong EK, Prokunina-Olson L, Wong WH, Lau CS, Chan TM, Alarcón-Riquelme M, et al. A new haplotype of PDCD1 is associated with rheumatoid arthritis in Hong Kong Chinese. Arthritis \& Rheumatism 2005; 52: 105862.

24. Iwamoto T, Ikari K, Inoue E, Toyama Y, Hara M, et al. Failure to confirm association between PDCD1 polymorphism and rheumatoid arthritis in a Japanese population. J Hum Genet 2007; 52: 557-60.

25. Lee SH, Lee YA, Woo DH, Song R, Park EK, et al. Association of the programmed cell death 1 (PDCD1) gene polymorphism with ankylosing spondylitis in the Korean population. Arthritis Res \& Therapy 2006; 8: R163.

26. Salama AD, Chitnis T, Imitola J, Ansari MK, Akiba H, et al. Critical role of the programmed death-1 (PD-1) pathway in regulation of experimental autoimmune encephalomyelitis. J Exp Med 2003; 198: 71-8.

27. Mataki N, Kikuchi K, Kawai T, Higashiyama M, Okada Y, et al. Expression of PD-1, PD-L1 and PD-L2 in the liver in autoimmune liver diseases. Am J Gastroenterol 2007; 102: 302-12.

28. Oikawa T, Takahashi H, Ishikawa T, Hokari A, Otzuki $\mathrm{N}$, et al. Intrahepatic expression of the co-stimulatory molecules programmed death-1 and its ligands in autoimmune liver disease. Pathol Int 2007; 57: 485-92.

29. Juran BD, Atkinson EJ, Schlicht EM, Fridley BL, Petersen GM, Lazaridis KN. Interacting alleles of the coinhibitory immunoreceptor genes cytotoxic T-lymphocyte antigen 4 and programmed cell-death 1 influence risk and features of primary biliary cirrhosis. Hepatology 2008; 47: 563-74.

30. Fife BT, Guleria I, Gubbels Bupps M, Eagar TN, Tang Q, et al. Insulin-induced remission in new-onset NOD mice is maintained by PD-1-PD-L1 pathway. J Exp Med 2006; 203: 2737-47.

31. Wang J, Yoshida T, Nakaki F, Hiai H, Okazaki T, Honjo T. Establishment of NOD-Pdcd1 -/- mice as an efficient animal model of type 1 diabetes. PNAS 2005; 102: 11823-8.

32. Asad S, Nikamo P, Torn C, Landin-Olsson M, Lernmark A, Alarcón-Riquelme M, et al. No evidence of association of the PDCD1 gene with type 1 diabetes. Diabet Med 2007; 24 (12): 1473-77.

33. Nielsen C, Hansen D, Husby S, Jacobsen BB, Lillevang ST. Association of a putative regulatory polymorphism in the PD-1 gene with susceptibility to type 1 diabetes. Tissue Antigens 2003; 62: 492-7.

34. Ni R, Ihara K, Miyako K, Kuromaru R, Inuo M, Kohno $\mathrm{H}$, Hara T. PD-1 gene haplotype is associated with the development of type 1 diabetes mellitus in Japanese children. Hum Genet 2007; 121: 223-32.

35. Hiromine Y, Ikegami H, Fujisawa T, Nojima K, Kawabata Y, et al. Trinucleotide repeats of programmed cell death-1 gene is associated with susceptibility to type 1 diabetes mellitus. Metabolism 2007; 56 (7): 905-9.

36. Bingley PJ, Bonifacio E, Mueller PW. Diabetes Antibody 
Standardization Program: first assay proficiency evaluation. Diabetes 2003; 52: 1128-36.

37. Mori M, Yamada R, Kobayashi K, Kawaida R, Yamamoto $\mathrm{K}$. Ethnic differences in allele frequency of autoimmunedisease-associated SNPs. J Hum Genet 2005; 50 (5): 264-6.

38. Hiromine $\mathrm{Y}$, Ikegami H, Fujisawa T, Kawabata Y, Noso S, et al. Molecular scanning of the gene for programmed cell death-1 (PDCD1) as a candidate for type diabetes susceptibility. Ann N Y Acad Sci 2006; 1079: 285-8.
39. Riley J, June C. The road to recovery: translating PD-1 biology into clinical benefit. TRENDS in immunology 2006; 28 (2): 48-50.

40. Vaarala O, Atkinson M, Neu J. The "Perfect Storm" for Type 1 Diabetes: The Complex Interplay Between Intestinal Microbiota, Gut Permeability, and Mucosal Immunity. Diabetes 2008; 57: 2555-62.

41. Round JL, Mazmanian SK. The gut microbiota shapes intestinal immune response during health and disease. Nat Rev Immunol 2009; 9: 313-24. 